\section{KN-8 Quasicrystals in soft condensed matter}

Ron Lifshitz ${ }^{1}$

1. Raymond \& Beverly Sackler School of Physics \& Astronomy, Tel Aviv University

email: ronlif@tau.ac.il

Recent years have shown a resurgence of scientific interest in quasicrystals, perhaps amplified by the award of the 2011 Nobel Prize in Chemistry to Dan Shechtman; yet mostly driven by breakthroughs in unlocking their crystal structure [1], and by the expanding scope of the field owing to the advent of a host of new experimental systems exhibiting aperiodic long-range order. Among these are soft-matter quasicrystals whose building blocks - rather than being individual atoms - are composed of large synthesized particles such as macromolecules, block co-polymers, nanoparticles, and colloids [2-5]. At these dimensions it is possible to design the interaction between particles, manipulate their positions, or even artificially place them at pre-assigned locations. It may also be possible to track the dynamics of individual particles, and in the optical domain even observe quantum wave functions. Consequently, these mesoscopic-scale quasicrystals provide rich and versatile platforms for the fundamental study of the basic physics of quasicrystals. At the same time they hold the promise for new applications based on artificial or self-assembled nanomaterials with unique physical properties that take advantage of the lack of periodicity, such as novel photonic metamaterials.

After giving a brief overview of the rapidly expanding field of soft-matter quasicrystals, I shall demonstrate how a quantitative understanding of their thermodynamic stability [6] has given us the ability to control the self-assembly of a variety periodic and aperiodic soft-matter crystals (at the moment only on the computer) and has led to the numerical discovery of a novel phase-a so-called "cluster quasicrystal" [7]. If time permits, I shall describe the design of nonlinear photonic quasicrystals for optical frequency-conversion applications [8].

[1] Takakura, Pay Gomez, Yamamoto, de Boissieu, Tsai (2007) Nature Materials 6, 58.

[2] Zeng, Ungar, Liu, Percec, Dulcey, \& Hobbs (2004) Nature 428, 157.

[3] Hayashida, Dotera, Takano, \& Matsushita (2007) Phys. Rev. Lett. 98, 195502.

[4] Talapin, Shevchenko, Bodnarchuk, Ye, \& Murray (2009) Nature 461, 964.

[5] Mikhael, Roth, Helden, \& Bechinger (2008) Nature 454, 501 .

[6] Lifshitz \& Diamant (2007) Phil. Mag. 87, 3021; Barkan, Diamant, \& Lifshitz (2011) Phys. Rev. $B$ 83, 172201.

[7] Barkan, Engel, \& Lifshitz (2014) Phys. Rev. Lett. 113, 098304.

[8] Lifshitz, Arie, \& Bahabad (2005) Phys. Rev. Lett. 95, 133901.

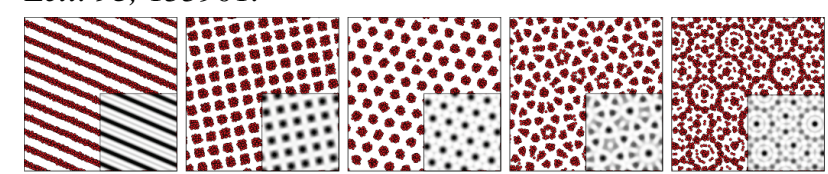

Figure 1. Self-assembled cluster crystals. Red particles represent molecules. Gray-scale images display the same structures, as predicted by mean-field theory. From left to right: stripes, 4-fold and 6-fold periodic crystals, 10-fold and 12-fold quasicrystals. Reprinted from Ref. [7].

Keywords: quasicrystals, aperiodic crystals, soft matter, macromolecules, nanoparticles, block co-polymers, colloids, self assembly, photonic crystals, metamaterials 